\title{
In-situ EM Characterization of Li-ion Battery through Multiple Cycles
}

\author{
Shen J. Dillon ${ }^{1}$, Kyong Wook Noh ${ }^{2}$ \\ 1. Materials Science and Engineering, University of Illinois Urbana-Champaign, Urbana, IL \\ 2. Department of Chemical Engineering, University of Illinois Urbana-Champaign, Urbana, IL
}

Cycle life often determines the commercial viability of a particular electrode chemistry or structure. The dramatic structural, crystallographic, and chemical changes that may occur in and around Li-ion battery electrodes can severely affect their cycle life. This provides a clear motive for developing an improved understanding of the nature of chemical and structural changes associated with electrochemical cycling. The sensitivity of reduced $\mathrm{Li}$ and commercial Li-ion electrolytes to air makes in-situ investigations imperative. This fact has driven a major push to characterize Li-ion cycling in-situ at the nanoscale using a variety of techniques. The spatial and temporal resolution associated with transmission electron microscopy is well suited to investigate battery electrodes and underlies the recent push to investigate associated materials in-situ. [1-9]

A variety of approaches have been pursued to characterize insertion and extraction of $\mathrm{Li}$, or $\mathrm{Na}$, via in-situ TEM. The most popular approaches have been direct reaction with partially oxidized Li or electrochemical cells based on vacuum stable ionic liquids.[7] Substantial effort has also focused on characterizing solid-state batteries[10-12] and batteries in commercial liquid electrolyte constructed within environmental cells[1, 8, 13]. Despite the large number of recent in-situ TEM studies, few consider multiple cycles. Understanding what occurs beyond the first cycle is particularly important because major losses in capacity typically occur after many cycles and it is not clear that the processes observed during the first cycle are necessarily representative. The ionic liquid, solid-state, and environmental cell approaches are chemically reversible and amenable to multiple cycles. Complete cycles occur at frequencies measured in hours making multiple cycles difficult to observe in electron microscopes that are typically shared instruments. An alternative approach is to perform multiple cycles and observe individual cycles in-situ intermittently.

This work characterizes lithiation of a $\mathrm{Sn}$ electrode after 4 cycles ex-situ performed within the environmental cell containing EC-DMC $1 \mathrm{M} \mathrm{LiPF}$. The emphasis is on testing the viability of the approach and determining whether the behavior observed after multiple cycles is consistent with what is observed during the first cycle. Figure 1 shows a time-lapse sequence taken during Li extraction at a constant voltage of $2 \mathrm{~V}$ versus Li metal. The response differs dramatically from first cycle results for the same system.[8] During the first cycle pores due to the volume strain associated with dealloying. After several cycles, Li extraction appears to promote the growth of a new phase (see arrows in Figure 1). X-ray diffraction studies suggest that $\mathrm{Sn}$ can partially amorphize during cycling and that microcrystalline Sn will convert to nanocrystalline Sn. It appears that the initially lithiated Sn electrode is partially amorphous and that it is recystallizing during Li extraction. Similar behavior has already been reported in the Li-Si system.[7]

\section{References:}

[1] Z Zeng, et al., Nano Lett. (2014) Ahead of Print.

[2] LQ Zhang, et al., Nanotechnology 24 (2013) p. 325702, 5

[3] W Liang, et al., ACS Nano 7 (2013) p. 3427-3433. 
[4] S Lee, et al., J. Phys. Chem. C 117 (2013) p. 24236-24241.

[5] MT McDowell, et al., Adv Mater 24 (2012) p. 6034-41.

[6] M Gu, et al., ACS Nano 6 (2012) p. 8439-8447.

[7] XH Liu and JY Huang, Energy Environ. Sci. 4 (2011) p. 3844-3860.

[8] KW Noh and SJ Dillon, Scr. Mater. 69 (2013) p. 658-661.

[9] CM Wang, et al., J. Mater. Res. 25 (2010) p. 1541-1547.

[10] D Ruzmetov, et al., Nano Lett. 12 (2012) p. 505-511.

[11] K Yamamoto, et al., Angew. Chem., Int. Ed. 49 (2010) p. 4414-4417, S4414/1-S4414/3.

[12] A Brazier, et al., Chem. Mater. 20 (2008) p. 2352-2359.

[13] M Gu, et al., Nano Lett. 13 (2013) p. 6106-6112.

[14] The authors are grateful for funding provided by the U.S. Department of Energy, Basic Energy Sciences (Contract No. DE-SC0006509).

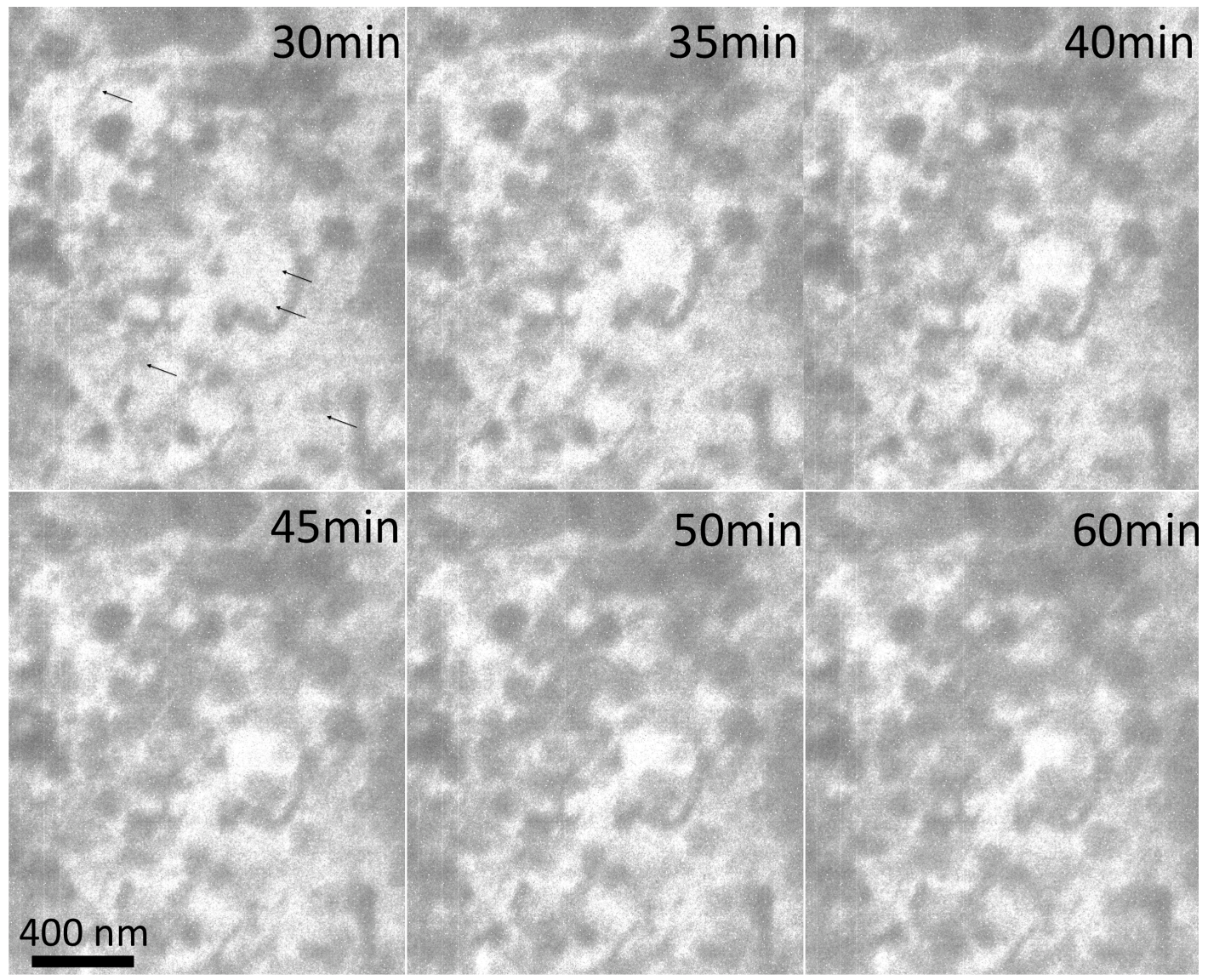

Figure 1. Bright-field TEM of Sn-Li thin film being delithiated at $2 \mathrm{~V}$ versus Li during the fourth cycle. The arrows in the image indicate regions that demonstrably decrease in intensity during the delithiation process. This is hypothesized to correlate with recrystallization of amorphous $\mathrm{Li}-\mathrm{Sn}$ when $\mathrm{Li}$ is extracted. 